\title{
ÊTRE DEBOUT : HISTOIRE DE LA FORMATION D'UN DES PRÉDICATS DE POSITION
}

\author{
ELIZAVETA BABAIEVA \\ Université d'État Lomonossov de Moscou, Russie
}

\begin{abstract}
Résumé. Larticle traite de l'histoire de la formation du predicat 'stand' dans la langue française. Depuis les temps anciens, le prédicat de 'standing' fait partie de deux oppositions: 'stand' VS 'sit', 'lie', (position correcte dans l'espace), 'stand' VS 'move'. Dans les langues romanes, l'acception 'standing' peut être transmise de plusieurs manières, par exemple, it. stare, roum. sta, esp. estar di pie, catal. estar dret, mais uniquement en français elle est exprimée par le prédicat être debout. L'article formule une hypothèse concernant les conditions sémantico-syntaxiques d'apparition et de distribution de ce prédicat, ainsi que sa chronologie.
\end{abstract}

Mots-clés : langues romanes et slaves, sémantique historique, histoire des idées, grammaire et sémantique

\section{INTRODUCTION}

Dans le cadre des approches fonctionnelles de la grammaire, les verbes de position dans l'espace (ou les verbes positionnels, angl. stance verbs) sont définis comme ceux qui requièrent un actant de lieu ou permettent diverses expressions de lieu. Les verbes de position dans l'espace sont bien étudiés dans une perspective typologique (v. par exemple dans Newman 2002). Comme le démontrent de multiples recherches, les langues diffèrent dans la manière de coordonner la localisation dans l'espace et le sujet de cette localisation. On oppose d'habitude les langues qui n'ont pas de prédicat locatif particulier à celles qui ont un prédicat verbal spécifique aux constructions locatives. Dans le premier cas, on distingue les langues qui n'ont pas de prédicat locatif du tout et les langues qui utilisent dans les constructions locatives une copule existentielle sans sémantique locative. La typologie de l'expression de la localisation statique reconnaît aussi différents types de systèmes : l'existence d'une copule unique (le verbe être) ou de systèmes d'expressions multiples. Entre ces derniers on peut distinguer des cas relativement simples avec quelques verbes de position (dit souvent de posture), comme, par exemple, le russe avec ses 4 prédicats (stojat' position 'debout', sidet' position 'assis', lezhat' position 'couché'/'étendu' et viset position '(sus)pendu') 
et des cas beaucoup plus complexes avec des verbes de position qui se comptent parfois par centaines. Les systèmes intermédiaires sont présentés par les langues amérindiennes, comme le démontrent des recherche de Colette Grinevalde (2005).

\section{ÊTRE DEBOUT : LE SENS ET SES EXPRESSIONS DANS LES LANGUES ROMANES}

Les verbes de position désignant 'être debout', 'être assis' et 'être couché' font partie de la liste de Morris Swadesh (la version moderne de la liste compte plus de 200 mots), établie dans les années 1940-1950, retravaillée depuis et utilisée pour mesurer le degré de parenté entre deux langues, c'est-à-dire du lexique de base, celui qui résiste le plus au changement. La racine indoeuropéenne ${ }^{*} s t a \overline{-}$ : ${ }^{*}$ stă- : ${ }^{*}$ stz-, qui en fait partie, est à la base de tout un vocabulaire, présenté dans les langues indoeuropéennes. Selon le linguiste russe V. Toporov (1996:27), cette racine est à l'origine de nombreux verbes-clés formant la base conceptuelle des textes cosmologiques et anthropologiques, reconstruits à partir des fragments de traditions indo-européennes archaïques retrouvées. La particularité du prédicat signifiant 'être debout' consiste en la possibilité de former trois oppositions :

1) 'être debout' VS. 'être assis'ou 'être couché' (les postures de l'homme);

2) 'être debout' VS. marcher ou courir (la position statique VS. le déplacement);

3) 'être debout' VS. se lever (il est debout (l'état final) VS il se lève (action) / il s'est levé (le résultat de l'action).

Dans les langues romanes, le sens 'être debout' peut être rendu par les verbes qui se rattachent à la racine indoeuropéenne ${ }^{*} s t \bar{a}-:{ }^{*} s t a ̆-: ~{ }^{*}$ stว- (it. stare, roumain sta) ou par quelques locutions verbales contenant le mot pied ou l'adverbe avec le sens 'droit' :

it. essere in piedi, stare in piedi, rimanere in piedi, essere ritto ;

esp.estar di pie;

port. estar de pié; ficar de pé;

roum. sta pe picioare;

catal. estar dret.

Dans ce contexte le français avec ses prédicats être/se tenir debout, offre un cas particulier. Pour expliquer cette particularité il nous faut descendre bien loin dans l'histoire de la langue. Comme l'explique Benveniste, 'le "sens" d'une forme linguistique se definit par la totalite de ses emplois, par leur distribution et par les types de liaisons qui en resultent. En presence de morphemes identiques pourvus de sens differents, on doit se demander s'il existe un emploi ou ces deux sens recouvrent leur unite' (Benveniste, $1954: 251$ ). Notre objectif est donc d'analiser l'ensemble de contextes médiévaux et surtout des sources lexicographiques et méthodiques dans le but de retrouver le type d'emploi propice à cette innovation grammatico-sémantique. 


\section{PROPOSITIONS POUR L'ANALYSE}

Selon Le Lieutenant-Colonel de Thomasson (1938 : 10-11), auteur de plusieurs essais sur le vocabulaire français, le verbe être représente 'une curiosité' : pour fixer sa conjugaison complète il faut recourir aux trois radicaux. Tout d'abord, nous savons que le verbe être est issu du latin populaire essere. Ce verbe vient, à son tour, du latin classique esse, qui se rattache, comme le grec eimai, à une racine indoeuropéenne ${ }^{*} e s-{ }^{*}{ }^{*}$ - 'se trouver', fonctionnant à l'origine comme copule. Le passé determiné $f u$ - vient d'une autre racine indoeuropéenne - *bhew-: ${ }^{*} b h u$ 'croître' (on retrouve ce sens originel dans le verbe grec fuō). Enfin, les participes passé et présent de ce verbe (été, étant) ont été empruntés à l'ancien français ester, qui correspond au latin stare. Donc, dans la sémantique assez complexe de ce verbe on voit le croisement de trois idées : exstence, croissance, position verticale.

\section{1 ÉMERGENCE DE LA SYNONYMIE ÊTRE SUR SON ESTANT ET ETTRE DEBOUT}

Les premières attestations du verbe ester datent du XII ${ }^{e}$ siècle. Dans les textes datés du XIII ${ }^{e}$ au XV $\mathrm{XV}^{\mathrm{e}}$ siècle, on trouve des collocations droit et estant, estant ou assis. Le corpus d'exemples du Dictionnaire du Moyen français (DMF) présentant la position d'une personne permet de distinguer deux types de contextes :

1) désignant tout simplement la position verticale d'une personne (ester, ester sur pieds): Un honme a ensemble la vertu ou puissance de soy seoir et de soy ester (Nicole Oresme, Le Livre du ciel et du monde) ; Il prist le cors de Nostre Seigneur et vint jusques a la rive du flun Jourdain et vit cele fame qui s'estoit a l'autre rive (Jean de Vignay, La Vie de sainte Marie l'Egyptienne); Quel cose faisons nous chi, estans sus nos piés et nous refroidant? (Jean Froissart, Chroniques);

2) désignant la position verticale d'une personne comme une attitude, liée à l'état émotionnel particulier, à la mobilisation de toutes ses ressources internes, qui précède un acte sacré (comme, par exemple, une prière) ou un événement extrêmement important (rus. stojat' na molitve $=$ prier estant debout, stojat' za pravdu (défendre la vérité) ; ville en yestance (ville qui résiste), predstat' pered Bogom(comparaître devant Dieux) ; dans ce type de contextes, qui sont, d'ailleurs, assez nombreux, on trouve des locutions ester en droit, ester en jugement, c'est-à-dire se présenter valablement comme demandeur ou comme défendeur devant la justice, par ex. : Ledit Bureau comperra ceans au Vermendois prouchain pour ester à droit et respondre à ce que ledit d'Orleans voudra proposer et dire (Nicolas de Baye, Journal).

Estant, le participe présent du verbe ester dans ses emplois substantivés, signifie une 'position verticale', surtout dans les expressions être en (son) estant, être ou se tenir sur son estant: Messires Jehans Chandos, pour la dolour qu'il senti, ne se peut tenir en estant, mès chei à terre (Jean Froissart, Chroniques). 
Cependant, au XIV siècle, ces expressions entrent en concurrence avec les prédicats être ou se tenir debout.

Debout, le substantif comme l'adverbe, est dérivé du nom bout, le déverbal de bouter. Ce verbe, devenu archaïque après le $\mathrm{XVI}^{\mathrm{e}}$ siècle, est consideré comme un emprunt francique. Selon le Französisches Etymologisches Wörterbuch de Walter von Wartburg (FEW), sa sémantique primaire est associée à l'idée du coup, donc il signifiait : 'pousser, heurter, frapper' (v. un but dans l'ancien français 'un coup'; une boutée dans l'ancien et moyen français 'choc, poussé, attaque' ; d'une boutée 'd'un coup'). Dans les textes du XII e siècle, on relève aussi les verbes rebouter et débouter qui rendent l'idée d'agression ou de la réponse à l'agression : porter un coup, attaquer, riposter, repousser, rejeter.

Le sens 'porter un coup', comme le démontre d'ailleurs l'histoire du mot coup, est porteur d'un très grand potentiel de développement. Au Moyen Âge le verbe bouter est resémantisé, s'intègrant dans le champ lexical 'germer' (v. l'évolution pousser 'heurter' $\rightarrow$ pousser 'sortir de terre, germer'). Au XIV siècle le nom bout signifie dans beaucoup de contextes l'extrémité (d'un objet, d'un corps ou d'un espace)', par exemple d'une lance, d'un pont, d'un navire, d'une contrée, d'une rivière. L'objet, dont l'extrémité est qualifiée du bout, peut avoir un des deux axes d'orientation : vertical ou horizontal. En cas d'orientation verticale l'extrémité est interprétée comme le haut, permettant la constitution du prédicat être debout avec la sémantique de position verticale :

Je ne suis point des excessifs / Importuns, car j'ai la pépie, / Dont suis au venc comme un châssis /

Et debout ainsi qu'une espie (A Madame d'Aleçon Marguerite de Valois de Clément Marot, 1518).

Ainsi vers le XIV ${ }^{\mathrm{e}}$ siècle s'installe la synonymie entre être en son estant et être debout. On voit aussi paraître dans les textes de nouveaux prédicats comme mettre debout, se lever debout. Au cour du $\mathrm{XV}^{\mathrm{e}}$ siècle l'averbal estant reste pourtant très fréquent.

\section{DÉCOMPOSITION DE LA SYNONYMIE ÊTRE SUR SON ESTANT ET ÊTRE DEBOUT}

La situation change au $\mathrm{XVI}^{\mathrm{e}}$ siècle. On peut constater, par exemple, que Robert Estienne, lexicographe et imprimeur français (1503-1559), hésite: dans son Dictionnaire françois-latin, contenant les motz et les manières de parler françois, tournez en latin, publié en 1539, l'expression être en son estant n'est pas mentionnée, mais on trouve les expressions estre ou se tenir debout sur ses pieds et le verbe stare, alors que plus tard, dans le Dictionnarium latinogallicum de 1552, il traduit le verbe stare par estre debout sur ses pieds et estre en son estant. Apparemment le prédicat estre en son estant, même ayant franchi le seuil du XVIe siècle, sort peu à peu de l'usage, cédant la place aux expressions estre / se tenir debout. 
Il existe pourtant un texte qui permettrait de comprendre pourquoi ce processus de concurrence synonymique s'est résolu au profit du prédicat être debout. Il s'agit de l'ouvrage, intitulé L'éclaircissement de la langue française de John Palsgrave (vers 1480-1554), contemporain de Robert Estienne, grammairien et lexicographe anglais, chapelain d'Henri VIII et précepteur de Marie Tudor, sœur du roi, à laquelle il a enseigné le français à l'occasion de son mariage avec Louis XII. Publié en 1530 à Londres, ce livre, adressée à des Anglais voulant apprendre le français, est considéré comme la première grammaire de la langue française.

L'auteur explique que l'expression estre debout peut signifier 'se porter bien': lacouché de cy pres est elle encore [déjà] debout? La posture verticale symbolise donc la vie active dans son opposition à la maladie ou à la mort (v. rus. vosstat' posle bolesni, vosstat' iz mertvych ). On retrouve cette interpretation plus tard, par exemple, dans le Dictionnaire universel d'Antoine Furetière (1690) : cet homme a été logtemps alitté, mais maintenant il est debout. Et dans Le Dictionnaire de l'Académie française (1694) il est debout s'explique comme il se porte mieux. Dans ce sens-là, selon Robert Estienne, on peut remplacer estre debout par se tenir sur son estant. En revanche Palsgrave traduit I stand upon me foote pris comme l'affirmation de l'orientation verticale de l'homme (sur pieds) par Je suis debout.

Il reste pourtant l'action de se lever, de changer de position de l'horizontale à la verticale: I aryse upon my fete, as a man do the that lueth alonge on the grounde, or that is felled to the grounde. Dans ce type de contexte, selon l'auteur, on emploie d'habitude des expressions comprenant le mot debout: Je me lieue debout, je madresse debout, or je m'adresse sur mes pieds. En même temps Palsgrave avoue avoir entendu des gens dire dans de tels contextes ( $J$ fynde also used in the same sens) je madresse sur mon estant. Donc, au debut du XVI ${ }^{\mathrm{e}}$ siècle il y avait encore des gens qui employaient sur son estant à l'oral.

Enfin, le contexte marqué serait l'impératif (the imparative mode), caractérisé parfois, selon Palsgrave, par un ton arrogant et la rapidité de la réplique : spokyn to an enemy or in haste. Dans ce type de contextes, affirme l'auteur, on emploie le mot debout sans copule ou verbe (they saye debout only and live out the verbe). On trouve des permiers attestations de cet emploi à la fin du $\mathrm{XV}^{\mathrm{e}} \mathrm{s}$.

L'impératif Debout! n'exprime pas seulement l'ordre de se lever, mais aussi l'appel à l'action (v. Debout! les damnés de la terre! / Debout! les forçats de la faim! d' Eugène Pottier) ; v. orientational methaphors en termes de George Lakoff et Mark Johnson: 'GOOD IS UP gives an UP orientation to general well-being, and this orientation is coherent with special cases like HAPPY IS UP, HEALTH IS UP, ALIVE IS UP' (Lakoff, Johnson, 2003 : 18). Cette forme grammaticale permettant de rendre la valeur fondamentalement directive de l'imperatif d'une façon lapidaire donnerait apparemment des avantages concurrentiels aux prédicats qui contiennent le mot debout.

Cette forme est commentée par des lexicographes du XVII ${ }^{e}$ siècle. Philibert Monet, par exemple, dans son Invantaire de deux langues francoise et latine (1635) note: Debout, mot excitatif à se mettre sur pied, se lever sur debout, qu'on se leue 
[: Eïa, agite, surgite, erigite vos in pedes, ad operam accingite]. Un autre lexicographe français, François-Antoine Pomey, dans son Dictionnaire royal (1671) donne aussi cet exemple : Debout Leve toi. En 1690 Antoine Furetière dans son Dictionnaire universel souligne: Quand on éveille quelqu'un à la haste, on luy crie, Debout, debout; fut debout, il est grand jour. Dans le Dictionnaire de l'Académie française (1694) on lit: Absolument, Debout, pour dire Levez-vous.

En même temps il faut prendre en compte des rapports entre le verbe de position ester et le verbe d'existence estre. Le premier finit par s'affaiblir et se rapprocher du sens de être. Il a été dépouillé de ses deux participes étant et été au profit de être. Le verbe ester ne se maintient que dans la langue juridique : ester en justice, ester en jugement.

\section{EN GUISE DE CONCLUSION}

Ainsi on peut supposer que la concurrence synonymique entre être en son estant et le néologisme être debout qui s'est formée au XIV siècle a pris fin vers le milieu $\mathrm{du} \mathrm{XVI}^{\mathrm{e}}$ siècle. Un des facteurs déterminants du renforcement des prédicats être/ se tenir debout porte le caractère grammatico-sémantique : la forme de l'impératif Debout! utilisée à partir de la fin du $\mathrm{XV}^{\mathrm{e}}$ siècle, permet le mieux de rendre l'idée de mouvement vers le haut et en même temps le passage de la passivité à l'activité.

\section{RECONNAISSANCE}

Cette recherche a été financée par RFFI (Russian Foundation for Basic Research) dans le cadre du projet № 19-012-00291 (Elaboration du Dictionnaire Actif de la langue russe).

\section{REFERENCES}

Benveniste, E. (1954) Problèmes Sémantiques de la Reconstruction. Word, 10 (2-3) : 251-264. Dictionnaire du Moyen français (DMF). Disponible en ligne http://www.atilf.fr/dmf [Consulté le 19 juin 2019].

Estienne, R. (1539) Dictionnaire françois-latin, contenant les motz et les manières de parler françois, tournez en latin. Paris : R.Etienne.

Estienne, R. (1552) Dictionnarium latinogallicum. Disponible en ligne https:/ fr.wikisource.org/ wiki/Dictionarium_latinogallicum_-_Robert_Estienne,_1552 [Consulté le 19 juin 2019].

Furetière, A. (1690) Dictionnaire universel, contenant généralement tous les mots françois tant vieux que modernes, et les termes de toutes les sciences et des arts. V I-III. La Haye : A. et R. Leers. Disponible en ligne https://gallica.bnf.fr/ark:/12148/bpt6k50614b.image [Consulté le 19 juin 2019].

Grinevald, C. (2005) Vers une typologie de l'expression de la localisation statique : le cas des prédicats locatifs. Linguistique typologique (pp. 33-54). Paris : Presses universitaires.

Lakoff, G., Johnson, M. (2003) Metaphors we live by. London: The university of Chicago press. Le Dictionnaire de l'Académie françoise (1694) Vol. I-II. Paris : Chez la Veuve de Jean Baptiste Coignard.

Monet, Ph. (1635) Invantaire de deux langues francoise et latin. Lyon : Veuve Rigaud. 
Newman, J. (ed.) (2002) The Linguistics of Sitting, Standing and Lying. Amsterdam, Philadelphia : John Benjamins.

Palsgrave, J. ([1530] 2003) L'éclaircissement de la langue française. Texte anglais original avec traduction et notes de Susan Baddeley. Paris : Honoré Champion.

Pomey, F.-A. (1671) Dictionnaire royal augmenté, enrichie d'un grand nombre d'expressions élégantes, de quantité de mots françois nouvellement introduits, des termes des arts et de cinquante descriptions, comme aussi d'un petit traité de la vénerie et de la fauconnerie. Lyon : Chez Antoine Molin.

Thomasson, Lieutenant-Colonel de (1938) Les curiosités de la langue française. Paris : Librairie Larousse.

Toporov, V. N. (1996) Ob odnom iz paradoksov dvizhenia. Neskolko zametchanij o sverchempiritcheskom smysle glagola 'stojat', preimuschestvenno v specializirovannych tekstach. Koncept dvizhenia v jazyke i kulture [À propos d'un des paradoxes du mouvement. Quelques remarques sur la metasignification du verbe 'stojat', principalement dans les textes spécialisés. Concept du mouvement dans la langue et la culture] (pp. 7-89). Moskva : Indrik.

von Wartburg, W. (1968-1969) Französisches Etymologisches Wörterbuch (FEW). V. 15. Basel : Zbinden. Disponible en ligne http://www.atilf.fr [Consulté le 19 juin 2019].

\title{
ÊTRE DEBOUT : HISTORY OF THE FORMATION OF ONE OF THE POSTURE VERBS
}

\begin{abstract}
The article deals with the history of the formation of the predicate 'stand' in French. The predicate of 'standing' has been part of two oppositions since ancient times: 'stand' VS 'sit', 'lie' (proper position in space), 'stand' VS 'move'. In Romance languages, the meaning of 'standing' can be transmitted in several ways, for example, it. stare, roum. sta, esp. estar di pie, catal. estar dret, but in modern French in this sense the predicate être debout is used. The article formulates a hypothesis regarding the semantic-syntactic conditions for the appearance and distribution of this predicate, as well as about its chronology.
\end{abstract}

Key words: French, historical semantics, concept history, grammar and semantics

Elizaveta Babaieva (docteur ès lettres, maître de conférences au Département de Linguistique française) travaille à l'Université Lomonossov de Moscou. Ses recherches portent sur les langues romanes et slaves, la sémantique, la lexicographie et l'histoire de la linguistique. Courriel : llevidova@gmail.com 